http://dx.doi.org/10.21707/gs.v11.n04a03

\title{
INFLUÊNCIA DA HIDRATAÇÃO DESCONTÍNUA NA GERMINAÇÃO DE SEMENTES E NO CRESCIMENTO INICIAL DE PLÂNTULAS DE AMBURANA cearensis (Allemão) A.C. SM. (Fabaceae)
}

\author{
Amanda Pereira dos Santos ${ }^{1, *}$, Marcos Vinicius Meiado ${ }^{1}$
}

${ }^{1}$ Laboratório de Fisiologia de Sementes, Departamento de Biociências, Campus Professor Alberto Carvalho. Universidade Federal de Sergipe. Av. Olimpio Grande, s/n, Bairro Porto, Itabaiana, Sergipe, Brasil. CEP: 49510-200

* Autor para correspondência: amandadsantos.12@gmail.com

Recebido em 07 de novembro de 2016. Aceito em 3 de maio de 2017. Publicado em 30 de dezembro de 2017.

Resumo - A hidratação descontínua proporciona maior germinabilidade em menor tempo, com maior velocidade e sincronização, além de favorecer a produção de mudas mais resistentes que podem ser utilizadas em projetos de recuperação de áreas degradadas. O objetivo deste trabalho estudo foi avaliar o efeito dos ciclos de hidratação e desidratação (HD) na germinação das sementes e no crescimento inicial de Amburana cearensis (Allemão) A.C. Sm. (Fabaceae). As sementes foram escarificadas com lixa e submetidas a 0,1,2 e 3 ciclos de 15 horas de hidratação e 24 horas de desidratação. Para cada ciclo, 50 sementes foram utilizadas e distribuídas em 10 repetições. No final do experimento foram avaliados a germinabilidade, o tempo médio de germinação, a velocidade média de germinação, o índice de sincronização, o comprimento da parte aérea e da raiz, o diâmetro do caule, a biomassa radicular, a biomassa de caule e folha e o número de folhas e folíolos. A hidratação descontínua não influenciou nenhum dos parâmetros de germinação e crescimento inicial, com exceção do tempo médio de germinação. As sementes de A. cearensis mostraram uma germinação mais lenta após três ciclos de HD. Embora não tenha sido observada uma influência positiva da hidratação descontínua na germinação e no crescimento inicial de $A$. cearensis, essa técnica de envigoramento das sementes e das plântulas não deve ser descartada prematuramente para a produção de mudas dessa espécie. Isso ocorre porque os ciclos de HD podem favorecer o estabelecimento de mudas sob condições de estresse, quando estas mudas são transplantadas no ambiente e são influenciadas por fatores ambientais.

Palavras-chave: CaAtinga; Ciclos de Hd; Germinação de Sementes; Producão de Mudas.

INFLUENCE OF DISCONTINUOUS HYDRATION ON SEED GERMINATION AND INITIAL SEEDLING GROWTH OF AMBURANA Cearensis (Allemão) A.C. Sm. (Fabaceae)

Aвstract - Discontinuous hydration provides a greater germinability in a shorter time, with greater speed and synchronization, in addition to favouring the production of more resistant seedlings that can be used in projects of recovery of degraded areas. This study aimed to evaluate the effect of hydration and dehydration cycles (HD) on the seed germination and initial growth of Amburana cearensis (Allemão) A.C. Sm. (Fabaceae). Seeds were scarified with sandpaper and submitted to 0,1,2 and 3 cycles of 15 hours of hydration and 24 hours of dehydration. For each cycle, 50 seeds were used and distributed in 10 replicates. At the end of the experiment we evaluated germinability, mean germination time, mean germination rate, synchronization index, shoot and root length, stem diameter, root biomass, stem and leaf biomass and the number of leaves and leaflets. Discontinuous hydration did not influence any of the germination and initial growth parameters, except for the mean germination time. Seeds of $A$. cearensis showed slower germination after three HD cycles. Although a positive influence of the discontinuous hydration on germination and initial growth of $A$. cearensis has not been observed, this technique of seed and seedling invigoration should not be prematurely discarded for 
the production of seedlings of this species. This is because HD cycles can favour the establishment of seedlings under stress conditions, when these seedlings are transplanted in the environment and are influenced by environmental factors.

Keywords: CaAtinga; Hd Cycles; Seed Germination; SEedling Production.

INFLUENCIA DE LA HIDRATACIÓN DISCONTINUA EN LA GERMINACIÓN DE SEMILLAS Y EN EL CRECIMIENTO INICIAL DE plántulas de Amburana cearensis (Allemão) A.C. Sm. (Fabaceae)

Resumen - La hidratación discontinua proporciona mayor germinabilidad en menor tiempo, con mayor velocidad y sincronización, además de favorecer la producción de plántulas más resistentes que se pueden utilizar en proyectos de recuperación de áreas degradadas. En este estudio se objetivó evaluar el efecto de los ciclos de hidratación y deshidratación (HD) en la germinación de las semillas y en el crecimiento inicial de Amburana cearensis (Alleman) A.C. Sm. (Fabaceae). Se escarificaron las semillas con lija y se las sometieron a 0,1, 2 y 3 ciclos de 15 horas de hidratación y 24 horas de deshidratación. Para cada ciclo, se utilizaron 50 semillas distribuidas en 10 repeticiones. Al final del experimento se evaluaron la germinabilidad, el tiempo medio de germinación, la velocidad media de germinación, el índice de sincronización, la longitud de la parte aérea y de la raíz, el diámetro del tallo, la biomasa radicular, la biomasa de las hojas y el número de las hojas e de las hojuelas. No se observó una influencia positiva de la hidratación descontinua en ninguno de los parámetros de germinación y crecimiento inicial, con la excepción del tiempo medio de germinación. Se observó una germinación más lenta después de someter las semillas a tres ciclos de HD. Aunque no se ha observado una influencia positiva de la hidratación discontinua en la germinación y en el crecimiento inicial de $A$. cearensis, no se debe descartar prematuramente esta técnica de envigoramiento de las semillas y de las plántulas para la producción de esta especie. Esto se debe a que los ciclos de HD pueden favorecer el establecimiento de las plántulas bajo condiciones de estrés, cuando estas plántulas son trasplantadas en el ambiente y son influenciadas por factores ambientales.

Palabras clave: Caatinga; Ciclos de Hd; Germinación; Producción de Plántulas.

\section{INTRODUÇÃO}

Os eventos de precipitação nos ambientes áridos apresentam baixos índices pluviométricos e, assim, a disponibilidade de água no solo desses ambientes tem grande influência no inicio da germinação e no estabelecimento (Wilson e Witkowsky 1998), o que torna os eventos de embebição seguidos de germinação cada vez mais raros (Drubrovsky 1998). Para espécies que ocorrem nesses ambientes, a quantidade de água e o tempo que esta fica disponível no solo podem não ser suficientes para promover a germinação e a embebição das sementes pode não ser contínua. Assim, as sementes tendem a ser submetidas a ciclos de hidratação e posterior desidratação (HD), sem concluir o processo germinativo (Bai e Romo 1995, Dubrovsky 1998, Adams 1999).

Essa hidratação descontínua pode proporcionar às sementes um elevado índice de sobrevivência durante a dessecação, demonstrando que essas sementes podem apresentar uma memória hídrica, que se refere à habilidade que as sementes têm em manter as mudanças bioquímicas e fisiológicas induzidas pelo processo de embebição das sementes durante a fase de hidratação (Dubrovsky 1996, 1998, Meiado 2013). A hidratação descontínua também pode beneficiar o desenvolvimento de plântulas, que podem apresentar maior crescimento e serem mais tolerantes aos estresses abióticos (Meiado 2013). Assim, a memória hídrica seria importante na produção de mudas para restauração de aéreas degradadas na Caatinga, pois proporcionaria a produção de mudas mais vigorosas, que apresentariam uma menor taxa de mortalidade após serem transplantadas para o ambiente (Meiado 2013). 
O aumento crescente de áreas degradadas em todo Brasil tem estimulado o desenvolvimento de novas técnicas de recuperação, a fim de tornar o processo de produção mais eficiente do ponto de vista técnico e econômico (Moraes et al. 2013). Assim, para melhorar a qualidade das mudas, a utilização de sementes que passaram por ciclos de HD para a sua produção pode ser uma técnica simples e eficiente. Diante do exposto, o objetivo deste estudo foi avaliar o efeito do ciclo de HD na germinação e no desenvolvimento inicial de plântulas de Amburana cearensis (Allemão) A.C. Sm. (Fabaceae) destinadas à restauração de áreas degradadas da Caatinga.

\section{Material e Métodos}

\section{Espécie estudada e local de coleta}

Amburana cearensis, conhecida popularmente como imburana-de-cheiro, é uma espécie arbórea típica da Caatinga, muito utilizada na fabricação de móveis. Suas sementes apresentam propriedades medicinais que permite sua utilização na medicina popular. Além disso, a espécie também é recomendada para restauração de áreas degradadas (Rebouças 2009, Angelim et al. 2007). Apesar de não estar ameaçada de extinção, a imburanade-cheiro encontra-se entre as espécies de interesse para pesquisa e conservação por apresentar declínio populacional e também interesse econômico (Martinelli e Moraes 2013).

Para realização deste estudo, os frutos de imburana-de-cheiro foram coletados em uma área de Caatinga do município de Salgueiro, no interior do Estado de Pernambuco. As sementes foram beneficiadas manualmente e armazenadas em sacos plásticos e em uma temperatura de $5^{\circ} \mathrm{C}$ até o início do experimento que ocorreu seis meses após a coleta.

\section{Ciclos de bidratação e desidratação (HD)}

Para superação da dormência, as sementes de imburana-de-cheiro passaram por escarificação física com auxilio de uma lixa. Depois de escarificadas, as sementes foram submetidas a ciclos de HD, sendo 0, 1, 2 e 3 ciclos. O tempo de hidratação e desidratação foi calculado de acordo com a curva de embebição da espécie, sendo o tempo de hidratação equivalente à metade da fase I da embebição (15 horas) e o tempo de desidratação correspondendo a 24 horas de secagem à temperatura ambiente. Logo que passaram pelos ciclos de HD, as sementes foram transferidas para sacos de polietileno preenchidos com solo, sendo dez repetições de cinco sementes por ciclo, as quais foram avaliadas diariamente, por um período de 15 dias.

\section{Análise dos dados}

Após 60 dias da montagem do experimento foram calculados os parâmetros de germinação das sementes e de desenvolvimento de plântulas. Os parâmetros para análise da germinação foram a germinabilidade (\%), o tempo médio de germinação onde ti é o período desde o início do experimento até a enésima observação (dias) e ni é o número de sementes germinadas no tempo $i$ (não o número acumulado, mas o número correspondente à enésima observação)], a velocidade média de germinação $(v=1 /$ t $)$ e o índice de sincronização, onde fi é a frequência relativa da germinação (i.e., a proporção de sementes germinadas em intervalo)] de acordo com Ranal 
e Santana (2006). A emergência de plântulas foi o critério utilizado para se considerar a germinação. Para análise de desenvolvimento das plântulas foram mensurados o comprimento $(\mathrm{cm})$ e biomassa seca $(\mathrm{mg})$ da parte aérea e subterrânea, diâmetro do caule $(\mathrm{mm})$ e o número de folhas e de folíolos. A biomassa da parte aérea das plântulas foi dividida em caule e folhas.

Para o cálculo dos parâmetros de germinação foi utilizado o programa GerminaQuant 1.0 (Marques et al. 2015). Foi verificada a normalidade dos dados e a homogeneidade das variâncias através do teste Shapiro-Wilk e Levene, respectivamente (Zar 2010). Todos os parâmetros de germinação de sementes e de desenvolvimento inicial das plântulas foram comparados pela ANOVA one-way com teste de Tukey a posteriori. As análises estatísticas foram realizadas no programa STATISTICA 13.0 com índice de significância de 0,05 (Statsoft 2016).

\section{RESUltados}

A hidratação descontínua não influenciou nenhum dos parâmetros de germinação de sementes que foram mensurados, com exceção do tempo médio de germinação (Figura 1 e Tabela 1), onde as sementes de imburanade-cheiro apresentaram uma germinação mais lenta após passarem por três ciclos de HD. A germinabilidade das sementes da espécie estudada foi superior a $70 \%$, sendo estatisticamente igual em todos os tratamentos avaliados $(\mathrm{F}=0,7823 ; \mathrm{gl}=3 ; \mathrm{p}=0,5125$; Figura 1$)$.

Figura 1 - Germinabilidade (\%) de sementes de Amburana cearensis (Allemão) A.C. Sm (Fabaceae) submetidas a ciclos de hidratação e desidratação (HD).

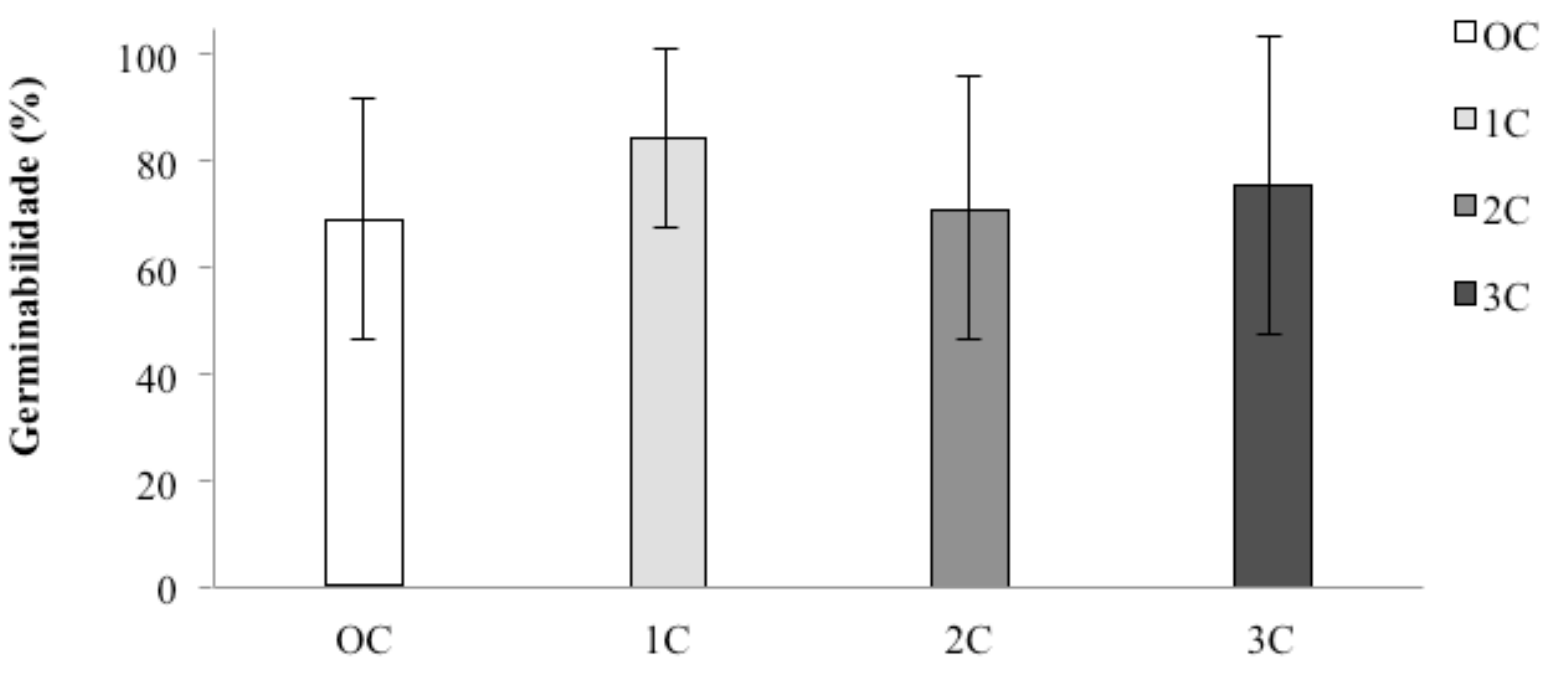

Ciclos HD

Os parâmetros de desenvolvimento inicial das plântulas de imburana-de-cheiro também não foram influenciados pela hidratação descontínua (Figura 2 e Tabela 1). As plântulas que foram formadas por sementes que passaram pelos ciclos de HD apresentaram comprimento similar da parte aérea e subterrânea após 60 dias de desenvolvimento (P.A.: $\mathrm{F}=0,3400 ; \mathrm{gl}=3 ; \mathrm{p}=0$,9393. P.S.: $\mathrm{F}=1,9050, \mathrm{gl}=3 ; \mathrm{p}=0,1462$; Figura 2). O diâmetro do caule, o número de folíolos e a biomassa de todas as partes das plântulas também não foram influenciados pela hidratação descontínua (Tabela 1). 
Tabela 1 - Parâmetros de germinação de sementes e de desenvolvimento inicial de plântulas de Amburana cearensis (Allemão) A.C. Sm (Fabaceae) submetidas a ciclos de hidratação e desidratação (HD).

\begin{tabular}{|c|c|c|c|c|c|c|c|}
\hline Parâmetro & OC & $1 \mathrm{C}$ & $2 \mathrm{C}$ & $3 \mathrm{C}$ & $F$ & g1 & $\mathrm{p}$ \\
\hline TMG & $10,6 \pm 0,8$ & $10,6 \pm 0,9$ & $10,0 \pm 0,7$ & $11,5 \pm 1,2$ & 3,860 & 3 & 0,0182 \\
\hline VMG & $0,09 \pm 0,01$ & $0,08 \pm 0,01$ & $0,09 \pm 0,01$ & $0,08 \pm 0,01$ & 2,879 & 3 & 0,0511 \\
\hline $\mathrm{Z}$ & $0,25 \pm 0,12$ & $0,34 \pm 0,15$ & $0,34 \pm 0,13$ & $0,36 \pm 0,12$ & 1,168 & 3 & 0,3372 \\
\hline $\begin{array}{l}\text { Diâmetro do } \\
\text { caule }\end{array}$ & $3,46 \pm 0,36$ & $3,55 \pm 0,58$ & $3,66 \pm 0,29$ & $3,94 \pm 0,48$ & 2,250 & 3 & 0,0991 \\
\hline$\underset{\text { raiz }}{\text { Biomassa da }}$ & $0,32 \pm 0,29$ & $0,24 \pm 0,16$ & $0,32 \pm 0,18$ & $0,26 \pm 0,11$ & 0,436 & 3 & 0,7280 \\
\hline $\begin{array}{l}\text { Biomassa do } \\
\text { caule }\end{array}$ & $0,12 \pm 0,09$ & $0,06 \pm 0,03$ & $0,07 \pm 0,04$ & $0,06 \pm 0,03$ & 0,735 & 3 & 0,0577 \\
\hline $\begin{array}{l}\text { Biomassa da } \\
\text { folha }\end{array}$ & $0,25 \pm 0,19$ & $0,17 \pm 0,08$ & $0,12 \pm 0,04$ & $0,14 \pm 0,10$ & 2,337 & 3 & 0,0899 \\
\hline $\mathbf{N}^{o}$ de folha & $6,0 \pm 0,6$ & $6,6 \pm 0,9$ & $6,4 \pm 0,8$ & $6,2 \pm 0,7$ & 0,984 & 3 & 0,0113 \\
\hline $\mathbf{N}^{\circ}$ de folíolos & $43,1 \pm 5,0$ & $45,6 \pm 5,6$ & $43,2 \pm 6,0$ & $42,7 \pm 7,1$ & 0,480 & 3 & 0,6983 \\
\hline
\end{tabular}

Figura 2 - Comprimento (cm) da parte aérea (P.A.) e subterrânea (P.S.) de plântulas de Amburana cearensis (Allemão) A.C. Sm (Fabaceae) submetidas a ciclos de hidratação e desidratação (HD).

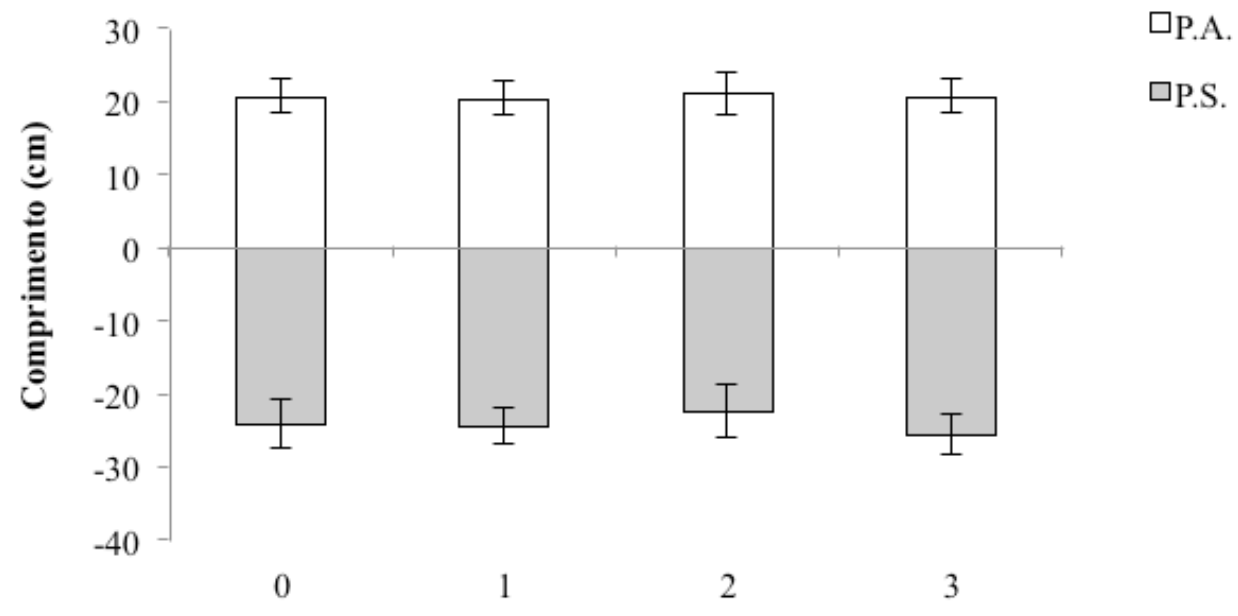

\section{Ciclos de HD}

\section{Discussão}

Embora não tenha sido observada uma influência positiva da hidratação descontínua na germinação e no desenvolvimento inicial de imburana-de-cheiro, essa técnica de envigoramento de sementes e plântulas não deve ser prematuramente descartada para a produção de mudas dessa espécie, pois ainda não se sabe sobre a 
sua influência em condições de estresse, quando as mudas são transplantadas no seu ambiente natural e sofrem a influência dos fatores ambientais.

Já existem evidências que, quando submetidas aos ciclos de HD, as plantas alteram sua fisiologia, tornandose mais vigorosas e tolerantes aos estresses abióticos em condições futuras, uma vez que as mudanças genéticas e bioquímicas ocorridas são passadas para futuras populações (Gamboa-de-Buen et al. 2006, Bruce et al. 2007). De acordo com Meiado (2013), os ciclos de HD podem favorecer diferentes fases do ciclo de vida das espécies arbóreas que produzem sementes na Caatinga e essa influência pode variar entre espécies. Espécies com germinação rápida, como é o caso da imburana-de-cheiro, podem ser favorecidas pela hidratação descontínua quando suas plântulas se desenvolvem sob condições não controladas no ambiente, mas a sua germinação não é influenciada pelos ciclos de HD (Meiado 2013). Já espécies com germinação lenta como, por exemplo, o licurí [Syagrus coronata (Mart.) Becc. (Arecaceae)] têm a germinação favorecidas pelos ciclos de HD, apresentando uma germinação mais rápida e sincronizada em campo, após serem submetidas aos tratamentos de hidratação de descontínua.

Assim, antes de se descartar a técnica da hidratação descontínua para a produção de mudas de imburanade-cheiro sugere-se que a sua influência seja avaliada no desenvolvimento de plântulas sob estresse controlado ou em condições de campo para que se tenha uma real noção dos benefícios que essa técnica pode trazer para a produção de mudas mais vigorosas que possam ser destinadas à recuperação de áreas degradadas na Caatinga.

\section{REFERÊNCIAS}

Adams R. 1999. Germination of Callitris Seeds in Relation to Temperature, Water Stress, Priming, and Hydration Dehydration Cycles. Journal of Arid Environments, 43(4):437-448.

Angelim AES, Moraes JPS, Silva JAB e Gervásio RCRG. 2007. Germinação e aspectos morfológicos de plantas de umburana de cheiro (Amburana cearensis) encontradas na Região do Vale do São Francisco. Revista Brasileira de Biociências, 5(2): 1062-1064.

Bai Y e Ramo JT. 1995. Seedling Emergence of Artemisia frigida in Relation to Hydration-Dehydration Cycles and Seedbed Characteristics. Journal of Arid Environments, 30(1): 57-65.

Bruce TJA, Matthes MC, Napier JA e Pickett JA. 2007. Stressful “memories" of Plants: Evidence and Possible Mechanisms. Plant Science, 173(6): 603-608.

Dubrovsky JG. 1996. Seed Hydration Memory in Sonorant Desert Cacti and Its Ecological Implication. American Journal of Botany, 83(5): 624-632.

Dubrovsky JG. 1998. Discontinuous Hydration as a Facultative Requirement for Seed Germination in Two Cactus Species of the Sonoran Desert. Jounal of the Torrey Botanical Society, 125(1): 33-39.

Gamboa-deBuen A, Cruz-Ortega R, Martínez-Barajas E, Sánchez-Coronado ME e Orozco-Segovia A. 2006. Natural Priming as an Important Metabolic Event in the Life History of Wigandia urens (Hydrophyllaceae) Seeds. Physiologia Plantarum, 128(3): 520-530. 
Marques FRF, Meiado MV, Castro NMCR, Campos MLO, Mendes KR, Santos OO e Pompelli MF. 2015. GerminaQuant: A New Tool for Germination Measurements. Journal of Seed Sciences, 37(3): 248-255.

Martinelli G e Moraes MA. 2013. Livro vermelho da flora do Brasil. Rio de Janeiro: Andrea Jakobsson Estúdio Editorial.

Meiado MV. 2013. Evidências de memória hídrica em sementes da Caatinga. In: Stelmann JR, Isaias RMS, Modolo LV, Vale FHA e Salino A. (Orgs), Anais do $64^{\circ}$ Congresso Nacional de Botânica: Botânica sempre viva. Belo Horizonte: Sociedade Botânica do Brasil, p. 89-94.

Moraes LFD, Assumpção JM, Pereira TS e Luchiari C. 2013. Manual técnico para a restauração de áreas degradadas no Estado do Rio de Janeiro. Rio de Janeiro: Jardim Botânico do Rio de Janeiro.

Ranal MA e Santana DG. 2006. How and Why to Measure the Germination Process? Revista Brasileira de Botânica, 29(1): 1-11.

Rebouças ACMN. 2009. Aspectos ecofisiológicos da germinação de sementes de três espécies arbóreas medicinais da caatinga. Recife: Universidade Federal Rural de Pernambuco.

Statsoft. 2016. STATISTICA 13.0.[homepage on the Internet]. StatSoft South America. [cited 2 April 2016]. Available from URL: http://www.statsoft.com.br

Wilson TB e Witkowsky ETF. 1998. Water Requirements for Germination and Early Seedling Establishment in Four African savana Woody Plant Species. Jounal of Arid Environments, 38(4): 541-550.

Zar JH. 2010. Biostatical Analysis. New Jersey: Prentice Hall Inc. 